\title{
Early outcome of combined endoscopic third ventriculostomy and choroid plexus cauterization in childhood hydrocephalus
}

\author{
Olufemi B. Bankole, FWACS, ${ }^{1}$ Omotayo A. Ojo, FWACS, ${ }^{1}$ Mathias N. Nnadi, FWACS, ${ }^{2}$ \\ Okezie 0. Kanu, FWACS, ${ }^{1}$ and John O. Olatosi, FWACS ${ }^{3}$
}

1Neurosurgery Unit, Department of Surgery; ${ }^{3}$ Department of Anaesthesia, Lagos University Teaching Hospital, Idi-Araba, Lagos; and 'Division of Neurosurgery, Department of Surgery, University of Calabar Teaching Hospital, Calabar, Nigeria

\begin{abstract}
OBJECT Although shunts have been the mainstay in treating hydrocephalus over the past 5 decades, the use of endoscopic techniques in addressing this disorder in children offers both the neurosurgeon and the patient a unique opportunity to avoid shunting and its attendant complications. The combination of endoscopic third ventriculostomy (ETV) with choroid plexus cauterization (CPC) remains uncommon in most centers despite its potential promise. The authors sought to investigate the efficacy of combining ETV and CPC (ETV+CPC) in treating childhood hydrocephalus in Nigeria. Infection and spina bifida contribute a high percentage of the cases of hydrocephalus in Nigeria.
\end{abstract}

METHODS Over a 2-year period, all children 0-18 years of age who had endoscopic treatment for hydrocephalus were prospectively evaluated to determine the need for subsequent treatment. Children who had the combination of ETV+CPC were identified as a subcategory and form the basis of this retrospective study.

RESULTS Twenty-two of 38 endoscopically treated children had undergone the combination of ETV+CPC for hydrocephalus of varied etiology. There was a male preponderance $(2.5: 1)$, and $90 \%$ of the patients were infants. The overall success rate was $75 \%$, with the best outcome in children with spina bifida. One child required a repeat ETV.

CONCLUSIONS The combination of ETV+CPC is useful in treating children with hydrocephalus of varied etiology. The complication profile is acceptable, and the overall success rate is comparable to that associated with shunt insertion.

http://thejns.org/doi/abs/10.3171/2014.10.PEDS14228

KEY WORDS endoscopic third ventriculostomy; choroid plexus cauterization; childhood hydrocephalus; outcome; developing countries

$\mathrm{H}$ YDROCEPHALUS remains a major contributor to the neurosurgical disease burden in Nigeria, as it does in many developing and more developed countries. ${ }^{11,25}$ In Nigeria, hydrocephalus mainly affects children, a reflection of the preponderance of young people in the population. While postinfective hydrocephalus (PIH) accounts for a major share of this condition, other congenital causes, such as neural tube defects, Dandy-Walker cysts, and aqueductal stenosis, are also commonly to blame. ${ }^{25}$ Because of a relatively poor socioeconomic status, most of these children do not always have ready access to special- ist care at the onset of the disease and typically can present weeks to months after symptom onset. ${ }^{6}$

The use of ventriculoperitoneal shunts, long regarded as standard treatment for hydrocephalus, is confounded in many less-developed nations because of its high complication rate, which is worsened by a health culture that lacks long-term follow-up by both the parents and the health care system. ${ }^{17,20}$ It is therefore expedient to look for a treatment option that is less prone to complications for children in our environment. ${ }^{6,15,25}$

Endoscopic third ventriculostomy (ETV) has become

ABBREVIATIONS CPC = choroid plexus cauterization; ETV = endoscopic third ventriculostomy; $\mathrm{MM}=$ myelomeningocele; $\mathrm{NPIH}=$ non-postinfective hydrocephalus; $\mathrm{PIH}=$ postinfective hydrocephalus.

SUBMITTED May 25, 2014. ACCEPTED October 6, 2014.

INCLUDE WHEN CITING Published online February 13, 2015; DOI: 10.3171/2014.10.PEDS14228.

DISCLOSURE The authors report no conflict of interest concerning the materials and methods used in this study or the findings specified in this paper. Dr. Bankole is the recipient of the PATH fellowship of CURE International to learn endoscopy at the CURE Children's Hospital, Mbale, Uganda. 
increasingly recognized as a viable treatment for hydrocephalus in children. ${ }^{21,26,27}$ It promises a simpler complication profile and lower risk of infection. Traditionally, ETV was reserved for children older than 2 years; more recently, however, its use in infants, especially when combined with choroid plexus cauterization (CPC), results in successful outcomes in many cases previously thought unsuitable for treatment by endoscopic means. ${ }^{23,26,27}$ At present, the scope of patients who can be successfully treated with endoscopic techniques is increasing. . $^{3,9,12,14}$

In light of this, we present our experience using the combination of ETV and CPC (ETV+CPC) in the treatment of children with hydrocephalus of varied etiologies. It has been suggested that combining these techniques may lead to better results in treating these children, who form a major part of our clinical practice. ${ }^{23}$

\section{Methods}

All children 0-18 years of age diagnosed with hydrocephalus and treated primarily with a combination of ETV+CPC over a 2-year period were prospectively recruited into a database and followed up. Consent was obtained from the parents or legal guardians of the patients, and the study protocol was approved by our hospital's ethics review committee. The decision to treat with ETV only or combined ETV+CPC was made using an algorithm described by Dr. Warf at the CURE Children's Hospital of Uganda. Using this algorithm, we categorized patients into postinfective hydrocephalus (PIH), non-postinfective hydrocephalus (NPIH), and myelomeningocele (MM) groups, according to a presumed or proven etiology, and treated them as outlined in Fig. 1.

All treated patients had symptomatic, progressive hydrocephalus and underwent imaging via transfontanelle ultrasonography or CT scanning. We do not routinely use MRI for patients with hydrocephalus given the prohibitive cost in our environment.

Only patients with at least 6 months of follow-up were included in this retrospective study. The procedure for the ETV+CPC was performed through a right frontal bur hole trajectory centered on the lateral angle of the anterior fontanelle, which was usually patent in most of these children. In older children, in whom the fontanelle was closed, the bur hole was placed just anterior to the coronal suture 2-3 $\mathrm{cm}$ lateral to the midline as described by other authors. ${ }^{2}$

A 2.8-mm flexible neuroendoscope (Karl Storz) was used for the procedure in all the patients. The use of a flexible endoscope made it possible to perform a complete bilateral CPC using just one bur hole. If the septum pellucidum is found intact, then a septostomy is performed to access the choroid plexus in the left lateral ventricle. We use a Bugbee monopolar electrocautery wire to fenestrate the floor of the third ventricle, as we do not have any Fogarty balloons. The Bugbee wire is typically used as a blunt dissection tool, although some cauterization of the floor of the third ventricle is sometimes required when the floor is thickened. The Bugbee wire is also used to coagulate the choroid plexus. Further details on the surgical technique for performing CPC have been reported elsewhere. ${ }^{23} \mathrm{In}$ using the flexible scope, our aim in the CPC is to cauterize

\section{Hydrocephalus Protocol}

\begin{tabular}{|l|l|l|l|l|}
\hline & A & B & C & D \\
\hline PIH & ETV + CPC & ETV+CPC & ETV & ETV+CPC \\
\hline NPIH & ETV+CPC & ETV+CPC & ETV+ CPC & ETV \\
\hline
\end{tabular}

PIH patients: after ETV, if cistern is scarred proceed with VPS placement and do not do CPC.

MM patients: if floor is thin and it looks easy, do ETV+CPC. Otherwise, place VPS.

\begin{tabular}{|l|l|l|}
\hline Type & AGE & AQUEDUCT \\
\hline A & $<1$ year & Open \\
\hline B & $>1$ year & Open \\
\hline C & $<$ 1 year & Closed \\
\hline D & $>1$ year & Closed \\
\hline
\end{tabular}

FIG. 1. Hydrocephalus protocol used in intraoperative decision making. Courtesy of Ben Warf. Published with permission. VPS = ventriculoperitoneal shunt.

all of the choroid plexus in both lateral ventricles including the temporal horn. The goal when performing an ETV is to expose the "naked" basilar artery and its branches posterior to the vertical leaf of Liliequist's membrane..$^{18}$

Success of the ETV+CPC procedure was determined after at least 6 months of follow-up. Endoscopic third ventriculostomy failure was defined as the need for any subsequent surgical procedure for CSF diversion or any mortality related to raised intracranial pressure or occurring in the postoperative period. ${ }^{26}$

\section{Results}

Fifty-three endoscopic procedures were performed over a 2-year period from July 2007 to June 2009 in children 0-18 years of age. Fifteen cases were abandoned (that is, only ventriculoscopy was done) for various reasons ranging from distorted anatomy to poor visibility. At that time, patients in these 15 cases proceeded to undergo placement of ventriculoperitoneal shunts or a reservoir device, as appropriate. Thirty-eight patients (72\%) underwent successful endoscopic surgery as the primary treatment for hydrocephalus. Of these, 22 patients underwent a combination of ETV+CPC. These 22 patients are the focus of this article.

There was a male preponderance among those treated using ETV+CPC, with a male/female ratio of 2.5:1. Twenty $(91 \%)$ of the 22 patients were younger than 1 year at the time of surgery (Fig. 2). The most common etiology group was the NPIH, while the PIH group was the least represented (Fig. 3). Two patients were lost to follow-up during the period under review and were thus excluded from the study. The follow-up period ranged from 6 to 27 months with a mean of 13 months and a median of 12 months.

Overall outcome at 6 months postprocedure revealed a 


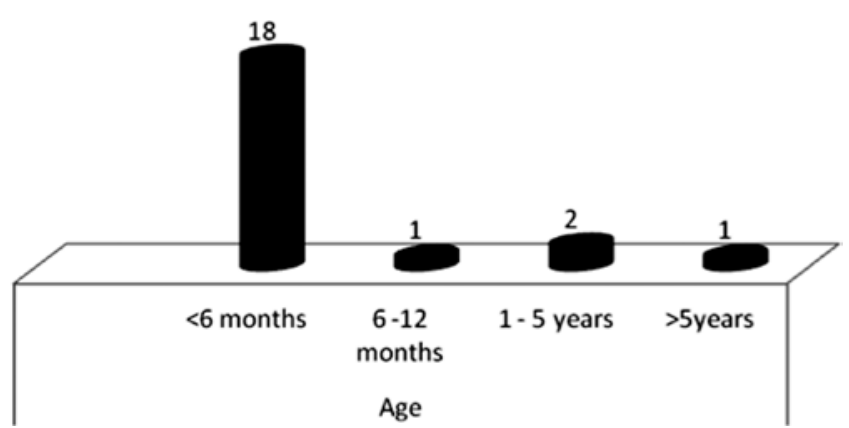

FIG. 2. Distribution of patients according to age at the time of surgery.

success rate of $75 \%$ ( 15 of 20 patients). If the 2 cases lost to follow-up were included as treatment failures, the rate decreased to $68 \%$. When the outcome was further categorized based on hydrocephalic etiology, the MM group had the best outcome of $87.5 \%$ while the PIH group had the worst outcome of 50\% (Table 1). Outcome did not appear to be related to age, although all of the treatment failures occurred in children who were younger than 6 months at the time of the procedure. In 1 child, the ETV procedure was repeated after initial clinical improvement; the stoma was found to be closed at the repeated endoscopy procedure and was reopened. Subsequently, the child was asymptomatic.

No significant intraoperative complications were noted in the study population. Bleeding encountered especially during CPC was easily controlled with irrigation and pressure. Most patients, however, had a bout of fever in the early postoperative period, which was self-limiting and easily corrected using paracetamol syrup $(10 \mathrm{mg} / \mathrm{kg})$. No patient had wound infection or sepsis related to the procedure.

\section{Discussion}

Hydrocephalus of varying etiology remains a major source of the neurosurgical disease burden worldwide. In developing countries such as Nigeria, the diagnosis is particularly burdensome to patients and their caregivers, as there is a lot of misunderstanding about the condition's diagnosis, etiology, and prognosis. Anecdotal evidence suggests a cultural tendency toward attributing the disease to the realm of the supernatural or the superstitious. ${ }^{6}$ When accompanied by a less than optimal health care system and a high default rate, traditional treatment options like shunt devices are fraught with many challenges.

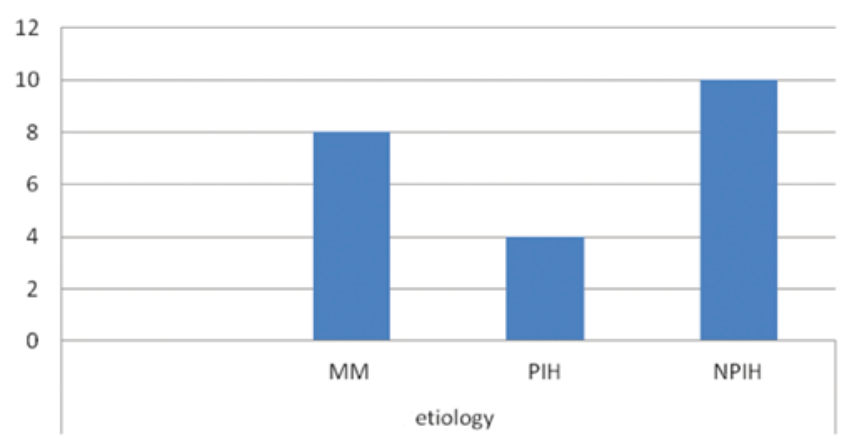

FIG. 3. Hydrocephalic etiology for the study population. The y-axis represents the number of patients. Figure is available in color online only.
While the use of endoscopic techniques does afford the neurosurgeon a treatment option less prone to complications, it does not do so by altering the coexisting social milieu. The many possible complications associated with shunt insertion have been well documented, and in an environment where prompt access to health care facilities and/or personnel is limited, the less the neurosurgeon is needed posttreatment the better. ${ }^{4,11,17,19,20}$

Another social advantage of endoscopic treatment is the ease with which it is accepted by parents. In our experience, many parents, while eager to accept shunt insertion when the child is ill, soon begin to pester the surgeon for shunt removal as soon as the child is perceived to be well. The presence of a shunt also becomes the culprit for any serious febrile episode the child has subsequently.

Prior to 2005, there were no reports in the literature on combining ETV and bilateral CPC, although earlier researchers had used both modalities at various times to treat patients with hydrocephalus., ${ }^{7,10,13,16,22}$ In reporting his experience in Uganda, Warf demonstrated that when ETV is combined with CPC a significant increase in the success rate is observed. ${ }^{23}$ This is particularly true for the category of patients with Chiari malformation Type II (post-MM) and PIH who have been thought unresponsive to endoscopic treatment. Since many children with hydrocephalus in our environment are likely to fall into this category, it is important to be able to offer them this treatment option. ${ }^{23}$

Many studies have been performed to evaluate the effectiveness of ETV in children, with varying success rates reported. Most of these researchers have used ETV alone as the treatment option. ${ }^{6,8,10,12,22}$ One of the authors (O.B.B.) received training in the use of combined ETV+CPC for treating hydrocephalus in 2006. Before this period, all patients with hydrocephalus had been treated with ventriculoperitoneal shunt insertion. Neuroendoscopic treatment began in July 2007 when the equipment became available. The aim of this study was to determine if the improved success rates reported by Warf when combining ETV+CPC could be achieved in our environment in patients with characteristics similar to those in Dr. Warf's patients.

Although the exact mechanism by which CPC improves outcome for ETV is unclear, several explanations have been offered for the observed benefit. ${ }^{5,24}$ In the series by Warf, the success rate ranged from $31 \%$ to $48 \%$ for children younger than 1 year with NPIH and PIH (with an open aqueduct) or with hydrocephalus due to MM treated using ETV alone. ${ }^{23}$ When this same category of patients had treatment with a combination of ETV+CPC, shunt placement was avoided in 62\%-73\%. Baldauf et al. reported a success rate of $43 \%$ in children younger than 2 years and $37.5 \%$ in infants after using only ETV. ${ }^{1}$ Yadav et al. achieved a clinical success rate of $83 \%$ using only ETV; however, most of the study population had aqueductal stenosis, which traditionally has the most favorable outcome. ${ }^{26}$ In our series, we were able to avoid shunting in $75 \%$ of the children treated with the combination of ETV and CPC (Table 1).

Postinfective hydrocephalus accounts for a large percentage of patients with hydrocephalus in many develop- 
TABLE 1. Overall success of ETV+CPC and success based on presumed etiology

\begin{tabular}{lcc}
\hline Etiology & No. of Patients w/ Good Outcome (total no.) & Percentage \\
\hline Overall & $15(20)$ & 75 \\
\hline MM & $7(8)$ & 87.5 \\
\hline NPIH & $8(10)$ & 80 \\
\hline PIH & $2(4)$ & 50 \\
\hline
\end{tabular}

ing countries. ${ }^{11,25}$ Even in patients with $\mathrm{PIH}$, a $50 \%$ reduction in shunt rates will have a significant impact on the quality of life as these children are able to avoid the longer term risks of shunt dependency.

Whether the benefits of this combined procedure are limited to children in developing nations has been debated. But this argument does not seem logical. While the circumstances in developing nations, as highlighted above and by other authors, make the ETV+CPC model particularly attractive, the benefits of shunt freedom should be no less so for patients in countries with better health care indices.

Although this study was based on outcome at 6 months postprocedure, no child, who at the time of evaluation for this study was assessed as being satisfactorily treated, has required any further treatment for hydrocephalus. It has been reported that most ETV failures occur soon after the procedure, usually within 6 months, and such early failure usually indicates an underlying unfavorable CSF pathophysiology. ${ }^{26}$

The adoption of endoscopic techniques to treat children with hydrocephalus at our center has seen a gradual but significant reduction in our shunting rates and subsequently in shunt complications, much to the delight of all concerned (Fig. 4).

\section{Conclusions}

In this preliminary study, we have found the combination of ETV and CPC to be a useful tool in treating childhood hydrocephalus of varied etiology, avoiding shunt placement in $75 \%$ of the study group. While a longer follow-up period is still recommended, this treatment modality should be carefully considered when evaluating children with hydrocephalus.

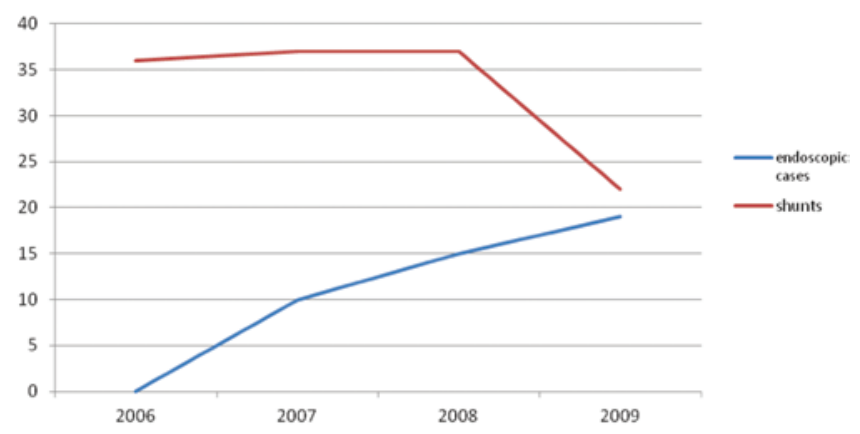

FIG. 4. Graph showing the decline in shunts inserted over a 3-year period. The $y$-axis represents the number of shunts; the $x$-axis, the years. Figure is available in color online only.

\section{References}

1. Baldauf J, Oertel J, Gaab MR, Schroeder HW: Endoscopic third ventriculostomy in children younger than 2 years of age. Childs Nerv Syst 23:623-626, 2007

2. Brockmeyer D: Techniques of endoscopic third ventriculostomy. Neurosurg Clin N Am 15:51-59, 2004

3. Brusius CV, Cavalheiro S: Endoscopic third ventriculostomy is a safe and effective procedure for the treatment of Blake's pouch cyst. Arq Neuropsiquiatr 71:545-548, 2013

4. Di Rocco C, Marchese E, Vellardi F: A survey of the first complication of a newly implanted CSF shunt devices for the treatment of nontumoral hydrocephalus. Cooperative survey of the 1991-1992 Education Committee of the ISPN. Childs Nerv Syst 10:321-327, 1994

5. Greitz D: Paradigm shift in hydrocephalus research in legacy of Dandy's pioneering work: rationale for third ventriculostomy in communicating hydrocephalus. Childs Nerv Syst 23:487-489, 2007

6. Idowu OE, Falope LO, Idowu AT: Outcome of endoscopic third ventriculostomy and Chhabra shunt system in noncommunicating non-tumor childhood hydrocephalus. J Pediatr Neurosci 4:66-69, 2009

7. Javadpour M, Mallucci C, Brodbelt A, Golash A, May P: The impact of endoscopic third ventriculostomy on the management of newly diagnosed hydrocephalus in infants. Pediatr Neurosurg 35:131-135, 2001

8. Jones RF, Stening WA, Brydon M: Endoscopic third ventriculostomy. Neurosurgery 26:86-92, 1990

9. Kamalo P: Exit ventriculoperitoneal shunt; enter endoscopic third ventriculostomy (ETV): contemporary views on hydrocephalus and their implications on management. Malawi Med J 25:78-82, 2013

10. Koch D, Wagner W: Endoscopic third ventriculostomy in infants of less than 1 year of age: which factors influence the outcome? Childs Nerv Syst 20:405-411, 2004

11. Komolafe EO, Adeolu AA, Komolafe MA: Treatment of cerebrospinal fluid shunting complications in a Nigerian neurosurgery programme. Case illustrations and review. Pediatr Neurosurg 44:36-42, 2008

12. Lee SH, Kong DS, Seol HJ, Shin HJ: Endoscopic third ventriculostomy in patients with shunt malfunction. J Korean Neurosurg Soc 49:217-221, 2011

13. Morota N, Fujiyama Y: Endoscopic coagulation of choroid plexus as treatment for hydrocephalus: indication and surgical technique. Childs Nerv Syst 20:816-820, 2004

14. Neils DM, Wang H, Lin J: Endoscopic third ventriculostomy for shunt malfunction: what to do with the shunt? Surg Neurol Int 4:3, 2013

15. O'Brien DF, Javadpour M, Collins DR, Spennato P, Mallucci CL: Endoscopic third ventriculostomy: an outcome analysis of primary cases and procedures performed after ventriculoperitoneal shunt malfunction. J Neurosurg 103 (5 Suppl):393-400, 2005

16. Pople IK, Ettles D: The role of endoscopic choroid plexus coagulation in the management of hydrocephalus. Neurosurgery 36:698-702, 1995

17. Prusseit J, Simon M, von der Brelie C, Heep A, Molitor E, Volz S, et al: Epidemiology, prevention and management of ventriculoperitoneal shunt infections in children. Pediatr Neurosurg 45:325-336, 2009

18. Rezaee O, Sharifi G, Samadian M, Haddadian K, Ali-Asgari A, Yazdani M: Endoscopic third ventriculostomy for treatment of obstructive hydrocephalus. Arch Iran Med 10:498503, 2007

19. Tamburrini G, Caldarelli M, Di Rocco C: Diagnosis and management of shunt complications in the treatment of childhood hydrocephalus. World Federation of Neurosurgical Societies. (http://www.wfns.org/pages/read_the_ 
reviews/97.php?rid=5\&jtype $=$ Old) $[$ Accessed December 30, 2014]

20. Teegala R, Kota LP: Unusual complications of ventriculo peritoneal shunt surgery. J Neurosci Rural Pract 3:361364, 2012

21. Teo C: Third ventriculostomy in the treatment of hydrocephalus: Experience with more than 120 cases, in: Hellwig D, Bauer BL (eds): Minimally Invasive Techniques for Neurosurgery. Berlin: Springer, 1998, pp 73-76

22. Teo C, Jones R: Management of hydrocephalus by endoscopic third ventriculostomy in patients with myelomeningocele. Pediatr Neurosurg 25:57-63, 1996

23. Warf BC: Comparison of endoscopic third ventriculostomy alone and combined with choroid plexus cauterization in infants younger than 1 year of age: a prospective study in 550 African children. J Neurosurg 103 (6 Suppl):475-481, 2005

24. Warf BC: Congenital idiopathic hydrocephalus of infancy: the results of treatment by endoscopic third ventriculostomy with or without choroid plexus cauterization and suggestions for how it works. Childs Nerv Syst 29:935-940, 2013

25. Warf BC: Hydrocephalus in Uganda: the predominance of infectious origins and primary management with endoscopic third ventriculostomy. J Neurosurg 102 (1 Suppl):1-15, 2005
26. Yadav YR, Jaiswal S, Adam N, Basoor A, Jain G: Endoscopic third ventriculostomy in infants. Neurol India 54:161-163, 2006

27. Zohdi AZ, El Damaty AM, Aly KB, El Refaee EA: Success rate of endoscopic third ventriculostomy in infants below six months of age with congenital obstructive hydrocephalus (a preliminary study of eight cases). Asian J Neurosurg 8: 147-152, 2013

\section{Author Contributions}

Conception and design: Bankole. Acquisition of data: Bankole. Analysis and interpretation of data: Bankole, Ojo. Drafting the article: Bankole, Nnadi. Critically revising the article: all authors. Reviewed submitted version of manuscript: all authors. Approved the final version of the manuscript on behalf of all authors: Bankole. Study supervision: Bankole.

\section{Correspondence}

Olufemi B. Bankole, Neurosurgery Unit, Department of Surgery, Lagos University Teaching Hospital, Idi-Araba, PMB 12003, Lagos, Nigeria.email: obbankole@cmul.edu.ng. 